\title{
Emerging Supercomputer Architectures
}

Paul C. Messina

California Institute of Technology ${ }^{\dagger}$

\section{DISCLAIMER}

\begin{abstract}
This report was prepared as an account of work sponsored by an agency of the United States Government. Neither the United States Government nor any agency thereof, nor any of their employees, makes any warranty, express or implied, or assumes any legal liability or responsibility for the accuracy, completeness, or usefulness of any information, apparatus, product, or process disclosed, or represents that its use would not infringe privately owned rights. Reference herein to any specific commercial product, process, or service by trade name, trademark, manufacturer, or otherwise does not necessarily constitute or imply its endorsement, recommendation, or favoring by the United States Government or any agency thereof. The views and opinions of authors expressed herein do not necessarily state or reflect those of the United States Government or any agency thereof.
\end{abstract}

I. Introduction

This paper vill examine the current and near future trends for commercially available high-performance computers with architectures that differ from the mainstream "supercomputer syste:ns in use for the last few years. These emerging supercomputer architectures are just beginning to have an impact on the field of high performance computing.

For some time it has been noted that sequential machines are approaching fundamental limits in speed imposed by the speed of light and heat transfer. This observation is coupled with the statement that significantly higher performance can only be achieved by decomposing a program into multiple parts and executing them concurrently on multiple hardware. Today's supercomputers achieve their performance through replication of certain components so that regular operations on vectors of operands can be performed at much higher speeds than operations on single pairs of operands. The well-known vector machines are of this type. Several of the vector computers also feature multiple CPUs, at present up to four, each with vector capability as well as the ability to access a large shared memory. In the context of this paper, these machines have "traditional" supercomputer architectures. Supercomputers manufactured in the United States include various Cray Research computers, Control Data

\footnotetext{
† Part of the preparation of this paper was performed at Argonne National Laboratory, the author's former employer. Work also supported in part by DOE grant DE-FG03-85ER25009 and DE-AC03-85ER40050, the Program Manager of the Joint Tactical Fusion Office, and the ESD division of the USAF. Also, grants from IBM, SANDIA and the Parsons and the System Development Foundations.
} 


\section{DISCLAIMER}

This report was prepared as an account of work sponsored by an agency of the United States Government. Neither the United States Government nor any agency Thereof, nor any of their employees, makes any warranty, express or implied, or assumes any legal liability or responsibility for the accuracy, completeness, or usefulness of any information, apparatus, product, or process disclosed, or represents that its use would not infringe privately owned rights. Reference herein to any specific commercial product, process, or service by trade name, trademark, manufacturer, or otherwise does not necessarily constitute or imply its endorsement, recommendation, or favoring by the United States Government or any agency thereof. The views and opinions of authors expressed herein do not necessarily state or reflect those of the United States Government or any agency thereof. 


\section{DISCLAIMER}

Portions of this document may be illegible in electronic image products. Images are produced from the best available original document. 
Cyber 205s, and the ETA-10. Several Japanese vendors (Fujitsu, Hitachi, and NEC) also manufacture vector supercomputers. Amdahl Corporation, a U.S. company, markets the Fujitsu line of vector supercomputers and NEC markets its vector computers in the United States. At present, Hitachi vector supercomputers are not marketed in the U.S.

Over the last few years, computers whose architectures feature a greater level of parallelism than vector supercomputers have been introduced by commercial vendors. Some have modest performance at this time but promise to increase dramatically in capability. Others already have potential performance that would put them in the supercomputer category. It is these systems that are the topic of this paper. While none of them is being used as a generalpurpose supercomputer today, there are signs that within a year or two that will change. This paper will focus on commercially produced computers. A few computers that are under development as research projects will be mentioned where it seems appropriate, but most architectures that are still at the basic research stage will not be discussed here.

\section{Characteristics of Today's Supercomputers}

To set the stage for examining the emerging supercomputer systems, we first survey briefly the present crop of supercomputers. In this paper, the term supercomputer will be used to mean the currently fastest general-purpose scientific computer. Performance is usually defined in terms of arithmetic operations on 64-bit operands. In practice, several systems will be called supercomputers at any point in time. It is not possible to say unequivocally that one machine is faster than all others. This is due to the difficulty of measuring performance for these machines and the inescapable fact that even the "general-purpose" systems will perform much better on some problems than on others. 


\section{Supercomputer Software Environment}

In addition to possessing the highest peak and achievable performance, today's supercomputers are expected to have operating systems and software environments that are sufficiently mature to allow productive use with moderate effort on the part of the user. The operating systems and related peripheral hardware must have high performance $1 / 0$ capability to match the internal speeds of the machine. The OS permits both interactive and batch use and coordinates the simultaneous access of the system by hundreds of users. Software and hardware for high-speed network connections are available to connect the supercomputer with a variety of front-end systems and computer networks. Optimizing compilers for popular languages such as Fortran and occasionally $\mathrm{C}$ are available. The Fortran compiler is expected to perform a substantial amount of vectorization automatically. Libraries of graphics and mathematical routines are available, as are many application programs such as structural analysis and circuit design packages.

Before the emerging supercomputers can be considered seriously as viable alternatives to the traditional variety, their software environment must offer the above capabilities. Mere peak performance is not sufficient to qualify a system as a supercomputer, especially if its architecture is novel.

\section{Supercomputer Performance Levels}

What then is the level of performance that must be achieved to merit the designation of supercomputer? Peak speeds of systems like the Cray 2 approach two billion floating point operations per second (abbreviated Gigaflops, or more commonly Gflops). A few user programs have achieved over one Gflop. Perhaps the first well-known instance of this was accomplished on a Cray 2 using all four processors simultaneously on one program [1]. Nearly that level of performance has been obtained on an Amdahl 1400 with four vector units and a 
NEC SX-2. The Amdahl 1400 achieved 940 Mflops on a two-dimensional filtering code used in seismic applications. This is noteworthy because the code performs a considerable amount of $\mathrm{I} / \mathrm{O}$ to disk (18.75 $\mathrm{MB}$ in and out) and the timing runs were done while the system was running other production jobs. The same machine ran order 1000 64-bit matrix multiply at 1046 Mflops. Few user programs approach those speeds, but performance of 50 million floating point operations per second (Mflops) per CPU is fairly common. In the next three years traditional supercomputers are expected to have basic cycle times that are one half to one quarter of today's fastest systems and the number of processors in one system will grow from four to sixteen or perhaps as many as sixty-four.

With those estimates in mind, we can say that peak rates of at least one Gflop and achieved performance on real user programs of several hundred Mflops are needed to qualify for the supercomputer designation today. Three to five years from now we should see peak speeds in the 10-30 Gflop range and achievable performance of several Gflops for a wide variety of scientific and engineering computations. These then are the performance levels to keep in mind when assessing the emerging supercomputer architectures against the traditional ones.

\section{Performance Measurement is Difficult}

A factor that complicates the measurement, and therefore the definition, of supercomputer performance is that the ratio of peak to achieved performance is growing larger. On a vector machine, a ratio of 10:1 might be seen. In highly parallel architectures the ratio between peak and minimum speeds achieved can be even more dramatic. On a parallel system with 1000 processors, each of which has vector hardware that is 10 times faster than the scalar

units, one could see a ratio of as much as 10,000:1 between peak performance and a worst case of single-thread code that did not vectorize or parallelize. To make matters worse, relatively small changes in the algorithm, its implementation, the source code, and the compiler 
can result in large changes in performance. When poor performance is experienced, it is difficult to determine whether it is due to inherent design flaws in the hardware or a minor aspect of the software that could easily be modified. Even when implementation inefficiencies are ruled out, it is foolhardy to assume that the system under study is not well suited for certain computations. Someone might invent a different algorithm for the same computation that uses the hardware effectively.

\section{New Definition of Supercomputer Performance Needed?}

As suggested by the following analysis, the emerging architectures may, perhaps surprisingly, play a significant role in the supercomputer arena even if their performance is an order of magnitude slower than the traditional supercomputer systems. Most traditional supercomputers are in centers with hundreds of users. A single user can expect to get only a small percentage of the available time. It is unusual for one group to get as much as $10 \%$ of the time on a supercomputer. If the cost of a computer is low enough that a small research group can buy one for its exclusive use and the performance of that system is $10 \%$ that of the "real" supercomputer, the same class of computations can be undertaken on the slower system, assuming that its memory and peripheral devices are adequate. It is indeed the case that some of the new systems offer sufticiently low cost and high performance to satisfy the scenario above. For example, at Argonne National Laboratory, Don Sinclair achieves for QCD computations two-thirds the performance of a Cray X-MP single processor on a Star Technologies ST-100 array processor. Both the Cray X-MP and the ST-100 programs are highly tuned. Since Sinclair has exclusive use of the ST-100, he can perform on that system computations of the same or larger magnitude as on a Cray X-MP. One series of computations [2] has consumed over 4500 hours of ST-100 time, which for this program is equivalent to 3,000 Cray X-MP hours. The elapsed time to do the computations is comparable or shorter than for using the Cray, given that it is extremely unlikely that a user can get exclusive access to a Cray X-MP for 
months at a time.

Therefore, some of the emerging high-performance systems will in effect change the operational definition of supercomputer performance levels. We may well need to start thinking of flops available per year to a single user or group as the measure of supercomputer capability.

\section{What are the Emerging Architectures?}

Computer architectures have evolved in several directions in the never-ending quest for speed and price-performance. Most of the architectural innovations are based on the strategy of concurrency. If several tasks can be performed at the same time, in parallel, then the overall problem can be solved in less time than if only one task is active at any point in time, given components of the same speed.

\section{A Look Backwards}

To put the emerging architectures into perspective, it will be helpful to examine briefly the history of architectural innovations aimed at high performance. In the 1960's high performance systems began to feature concurrency. Processing of instructions by the central processing unit was decomposed so that one instruction could be fetched from memory while another instruction was decoded and a third was executcd. Floating point arithmetic operations are complicated and therefore are often much slower than other operations. For scientific computation, floating point operations often dominate the execution time, so there is considerable incentive to speed them up. In addition to instruction pre-fetching and decoding, floating point function units were decomposed into segments, each of which did part of the 
arithmetic operation. These segmented functional units operate in pipeline fashion. Once an operand has entered the functional unit and has been operated on by the first segment, a second operand can enter and begin processing in the first segment while the first operand is in the second segment of the functional unit, and so on. In addition, multiple arithmetic functional units began to emerge, so that, for example, two floating point additions/subtractions and one floating point multiplication could all occur at once.

Access to memory and input/output operations received similar attention. Memory units were split into "banks" so that successive data accesses, each one to a separate bank, could be carried out with considerable overlapping. Cache memories were added to exploit the locality of instruction and data access that many programs exhibit. These memories are much smaller but substantially faster than main memories. If program instructions and the data they require are in cache memory, they can be fed to the instruction processing unit much faster than if they must be fetched from main memory. Specialized I/O processors were introduced so that much of the effort associated with $\mathrm{I} / \mathrm{O}$ operations could be performed by the $\mathrm{I} / \mathrm{O}$ processor, leaving the central processor free to execute other program instructions.

The 1960's also saw the birth of several new types of architectures. These architectures used a different approach to gain performance. Rather than design a single processor and memory system with many elaborate features for concurrency, multiple processors were combined to form a single system. The must straightforward of these approaches was to have two or four entire CPUs share memory and peripherals. Each processor ran programs independently of the others, so there was no gain in speed for any one program, but a larger number of programs could be executed in a given period of time. That is, greater throughput was achieved. Large mainframes built by IBM and CDC were among the first to offer this type of configuration. In the 1980's this arrangement has propagated to minis and superminicomputers, for example many of DEC's 8000 series models.

Parallelism aimed at increasing the speed with which a single program could be processed became a topic of attention in the late 1960's and early 1970's. The ILLIAC IV 
employed 64 rather simple processors and connected them in such a way that they could work simultaneously on computations for the same program. In the ILLIAC IV, all processors operated the same instruction in lock step. Each processor had its own modest sized memory and could send messages to four neighboring processors. The ICL Distributed Array Processor (DAP) utilized a much larger number (4096) of processors, but each processor was extremely simple and had a small memory. Again one instruction at a time was issued, but all 4096 processors could execute (or ignore) it, each on its own data. (In the early 1980's the Goodyear Massively Parallel Processor went even further in this direction: it had 16,384 processors. We describe the DAP and MPP in more detail later in the paper.)

In the 1970's the trend of ever more elaborate single-processor systems continued. Vector machines such as the CDC Star, Cyber 203 and 205, and the Cray-1 employed the techniques for speeding up single processors mentioned earlier, but in addition had one or more pipelines that optimized the execution of regular operations on vectors of numbers. The Cyber 205 can have up to four such pipelines, so if several vectors can be operated on independently of each other, those operations can be performed simultaneously. These vector machines have become the dominant architecture for very high performance numerical computing. Today's supercomputers have the same basic architecture as the vector machines of the 1970's. Higher performance for a single vector system has been achieved through shorter basio cycle times and removing some bottlenecks. Für exauple, the Cray X-MP has three paths to memory that can be active simultaneously rather than one path; as in the Cray 1s.

The Denelcor Heterogeneous Element Processor (HEP) was a sophisticated pipelined MIMD system developed under funding from the Army Ballistics Research Laboratory and marketed as a commercial product in the early 1980s. The HEP consisted of one to sixteen Program Execution Modules (PEMs) that were connected to globally shared memory via a pipelined switch. Each PEM had local memory and a large register set. An eight-stage pipeline executed all instructions (except floating point divide) in eight clock cycles. The basic cycle time was 100 nanoseconds, so once the pipeline was full a result was produced every 
100 nanoseconds. Process creation and management (up to 64 per PEM) was done mostly in hardware. This design made possible the exploitation of very fine grain parallelism within a PEM, as well as larger grain decomposition among PEMs. The HEP was aimed at the supercomputer market but its initial implementation was not cost effective and software was immature. When Denelcor Corporation went bankrupt, there were four or five customer sites. Only one, Messerschmidt, used it for production work: controlling a flight simulator.

The supercomputers of the mid 1980's are vector machines. Systems from Cray and ETA feature two, four, or eight complete vector processors, all capable of accessing a large memory. In some cases there is a sizable memory that is local to each processor as well. The Japanese supercomputers are all single processor models but have optional multiple vector pipelines.

\section{The New Architectures}

It is difficult to devise a systematic taxonomy of computers with advanced architectures. Among the possible distinguishing features are:

- the mechanism for control of execution-program, dataflow, or demand-driven;

- $\quad$ sequential or parallel execution;

- $\quad$ single or multiple instruction streams (SIMD versus MIMD);

- homogeneous or heterogeneous processors;

- $\quad$ grain-size-the size of the units of work that can be performed in parallel;

- the method and topology of connecting processors to memory and processors to processors; 
- the characteristics of each processor-e.g., bit-serial, microprocessor, long instruction word, scalar, vector, or specialized;

The major architectural trends in commercially available machines are (1) programdriven multiprocessor systems featuring bus-oriented connections between processors and memory, (2) multiprocessor systems with hypercube connection schemes, (3) wide-word machines, and (4) lattice connected systems. Many systems combine several of these architectural features. In many cases vector processors are available. Soon several of the shared memory systems will have variants in which groups of processors that share memory are linked to each other and perhaps to a global shared memory. These designs are sometimes called "cluster architectures." Today's commercial multiprocessor systems are homogeneous. The processors used are generally not designed especially for use in parallel architectures; rather, they are processors that were developed independently for general-purpose use.

Many advanced architecture commercial systems are not initially designed or marketed to compete in the supercomputer arena. However, the designs can generally be extended and developed to the point that supercomputer performance could be achieved at least at the hardware level. Developing the rest of the characteristics of a true supercomputer is more problematic. For example, considerable time and money is necessary to develop the requisite software base and high performance I/O capability. It appears to be mostly a question of corporate strategy as to whether the systems with innovative architectures try to compete with the traditional supercomputers.

\section{Bus-connected Multiprocessors}

Bus-connected multiprocessor systems have been introduced by several commercial vendors. Most of these systems use one or more high-speed buses to connect relatively inexpensive processors of slow or moderate speed to a memory that is directly addressable (shared) by all the processors. The first generation of several of these systems uses NS 32032 or MC 
68020 processors. Encore's Multimax-1, Sequent Balance 21000, and Flexible Corporation's Flex/32 are examples of this type of system. Current models can house up to 30 processors, so their present processing power is on the order of a few Mflops. New models are being introduced this year that use faster microprocessors and other enhancements such as larger caches and faster buses. For example, the new Sequent Symmetry Series uses the Intel 80386, which is three to four times faster than the NS 32032 used previously. Even faster floating point performance will be available through the addition of a floating point option based on Weitek chips. With this option, each processor should achieve about $0.3-2.0$ Mflops; with perfect speed-up, a 30-processor system might deliver 20 Mflops on real applications. At that level, while still not a high performance numerical computation machine, it would be twenty times faster than a conventional supermini like a VAX 8800.

In contrast, Alliant has a higher performance system, the $F X / 8$, that includes up to eight custom vector processors and up to thirteen MC68020 microprocessors. The 68020 processors are used for non-computational tasks such as compilation, editing, and the operating system. The peak speed of the FX/8 with 8 vector processors is about 46 Mflops for 64-bit data. Typical performance is much lower, say about 10 Mflops, but that is impressive in that it rivals the supercomputers of a decade ago, such as the CDC 7600.

Systems of this type have proved to be cost effective alternatives to traditional superminis and minisupers, but are not in the supercomputer performance range. Bus connections cannot support very large numbers of processors, since the bus bandwidth must be shared by all the processors on the bus. Bus speeds become one limiting factor. However, the "clustering" approach can in principle yield systems with peak speeds in the supercomputer range. The Flex/32 is scalable to high performance configurations, in the sense that up to 1024 cabinets, each with 20 processors, can be linked together. In addition, there are research projects underway to link together other systems so that the resulting system will approach supercomputer speeds. The Cedar project at the University of Ilinois at Urbana, is developing a system that will connect up to 64 Alliant FX/8s. This configuration would have a peak speed 
of about 3 Gflops. The ULTRAMAX project, carried out jointly by Encore Corporation and Carnegie-Mellon University, will also use the strategy of connecting multiple clusters to achieve performance in the range of $10^{\circ}$ instructions per second (Gips). The clusters will consist of Encore Corporation's Multimaxes, each with 20 processors. It has not yet been demonstrated that one can get high performance for real applications on cluster systems, but experience with distributed memory machines suggests that it will be possible.

\section{Hypercube Architecture}

The second popular advanced architecture features the hypercube connection scheme. With this architecture, each processor has private memory and is connected to other memory/processor combinations (known as "nodes" in the parlance of message passing architectures) via a high speed channel. In some variations of this architecture, subsets of nodes share memory. This architecture scales well to large numbers of processors, because in a hypercube with $\mathrm{N}$ nodes each node is connected to only $\log _{2} N$ other nodes, where $\mathrm{N}$ must be a power of $2\left(N=2^{m}\right.$ for some $m$ ). As with bus-connected systems, most of the commercial offerings use rather slow microprocessors at each node. In early versions of these systems, the communication speed among processors has been so slow that it has further throttled the performance achieved.

Intcl markets the iPSC line of hypercube architecture systems. There are three types of configurations. The standard system has up to 128 nodes; at each node is an Intel 80286 microprocessor with the Intel 80287 floating point coprocessor and 0.5 Mbytes of memory. Each node is capable of about 0.03 Mflops. The large memory version has the same processors but with 4.5 Mbytes of memory. Its maximum configuration is 64 nodes. Finally, there is a version with vector processors at each node. This model is offered in configurations with up to 64 nodes each with 1.5 Mbytes of memory. Architecturally it is possible to build a vector system with 128 nodes, but that configuration is not a standard product. The peak speed of the 64-node configuration is 424 Mflops (64-bit), fast enough to approach supercomputer 
performance.

On a seismic modeling application, performance of 227 Mflops was achieved on a 32 node system with vector processors. The inner loop of the computation was microcoded by hand to achieve this performance. This computation was done with 32-bit accuracy.

The largest Intel vector system built to date has thirty-two nodes. A new model with faster node processors (Intel 80386) and more memory has been announced; each node will be about three times faster and new communications hardware and software should substantially increase the achievable performance. While the vector option will use the same board as at present and therefore will have the same peak speed, higher speeds should be possible on real application programs because of better communications and compilers. Fortran, C, and LISP are available.

A hypercube with up to 1024 processors, each approximately the speed of a VAX $11 / 750$, is available from NCUBE Corporation. Each node has either $128 \mathrm{~K}$ or $512 \mathrm{~K}$ bytes of memory. The processors are a custom design and extremely compact. Sixty-four processors fit on a single board. Even though there is no special hardware for floating point arithmetic or vector processing, the largest configuration has peak speeds of over a billion instructions per second and two hundred Mflops. It also has potential for high-speed $1 / O$ : eight $1 / O$ channels can transmit data at 90 Mbytes/second bidirectionally. As was noted earlier, an important characteristic of supercomputers has been the ability to do high speed $1 / O$. The NCUBE system achieves supercomputer performance on some computations. For a Monte Carlo photon transport program, a 64-node NCUBE ran at about the same speed as a Cray X-MP single processor for small problems and 1/12th the speed on large problems. The Cray code was vectorized. [3] Fortran and $C$ are available.

Floating Point Systems has introduced the T-Series, in which modules, each containing eight array processors, are connected to each other with the hypercube scheme. The T-Series can be configured with up to $2^{14}$ processors. Each processor has a peak speed of 16 Mflops. The T-Series thus is potentially the most powerful computer system available: peak 
performance of the maximum configuration is 262 Gflops. At present the only programming language available is Occam and the performance actually achieved is usually far short of the peak performance, due to its very slow scalar speed. However, on the biggest existing $T$ Series configuration, a 128-node system, hand-coded matrix multiplication routines have run at a speed of 1.2 gigaflops for matrices of order 1024. Two-dimensional convolution achieved similar speeds. Fortran and $C$ will become available in the near future.

Ametek Corporation also has announced systems with the hypercube architecture. Its initial model used Intel 80286/80287 processors; maximum configuration was 256 nodes.

The hypercube connection scheme is also used by a radically different system: the Connection Machine, built by Thinking Machines Corporation. The Connection Machine is massively parallel: its maximum configuration has 65,536 processors. They are quite different from the other processors discussed to date: each is a one-bit computer. In the original model, the CM-1, each processor has only 512 bytes of memory and floating point operations are performed by groups of processors (microcode is provided to do this). The Connection Machine Model 2 (CM-2) has 65,536 bit-serial nodes and 2048 floating-point units (based on a Weitek chip set). In the CM-2, each processor has $8 \mathrm{~K}$ bytes of memory. The system is built from chips that contain 16 bit-serial processors each. Within a chip all 16 processors are connected. Connections between the chips are in the hypercube topology. Unlike the other hypercubes, all of which are MIMD, the Connection Machine is an SIMD machine. All processors receive the same instruction each cycle, which they may ignore depending on the setting of a flag bit. The Connection Machine architecture is therefore similar to that of the DAP and MPP described below. They also use bit-serial processors and have the SIMD control model. A key difference is that the MPP and DAP use lattice connections whereas the Connection Machine uses the hypercube topology. The only languages currently available on the Connection Machine are special versions of LISP and C, but Fortran will be added in a few months. While the original Connection Machine was designed with artificial intelligence applications in mind, it can perform floating point arithmetic at high rates. Speeds of 50 
Mflops have been achieved on a 32,768 node configuration of the first model and it appears that 100 Mflops would be reached with a 65,536 node system. The CM-2 is claimed to achieve 2.5 Gflops for 64-bit matrix multiply and 5 Gflops for 64-bit dot product. This machine has a sophisticated programming environment but it is oriented to programming in an enhanced version of LISP, a language that is heavily used in artificial intelligence work but is virtually unknown in numerical programming. An enhanced version of $\mathrm{C}$ is also available.

Finally, although not a commercial product, the Mark IIIfp hypercube system (that Jet Propulsion Laboratory is building as a research project) is worth mentioning. The Mark IIIfp can be configured with up to 256 nodes. Each node has two MC 68020 microprocessors, one for computation and one for communications; four megabytes of memory; and a pipelined floating point unit with relatively high performance, currently estimated at 10 Mflops for 64bit arithmetic. The floating-point board uses the new Weitek XL series of chips. Preliminary timings on the 32-bit version of the chips on the Mark IIIfp board (the 64-bit version of the chips is not yet available) yielded speeds as high as 16 Mflops for a hand-coded assembler code to multiply two $3 \times 3$ complex matrices. This computational kernel is at the heart of many QCD computations. The 128-node configuration under construction will therefore have a peak speed of over one Gflop, putting it in the supercomputer performance level.

\section{Wide Instruction Word Machlnes}

It was noted in an earlier section that one technique for gaining higher performance in computers was to have more than one functional unit in the CPU and to find ways to utilize them concurrently. This approach takes advantage of parallelism at a very fine level, that of individual arithmetic or logical operations. Array processors of the 1970's exploited this approach, the Floating Point Systems product line being the most successful. On the FPS 164 and 264 attached processors, array indexing, loop counting, and data fetching from memory can be performed simultaneously with arithmetic operations. Wide instruction words are used 
to specify the simultaneous operations. Furthermore, up to 15 MAX (Matrix Algebra Accelerator) boards can be added to these machines. Each MAX board has two multipliers and two adders. Compiler technology did not yield code that took full advantage of the multiple units; therefore, intricate hand coding, sometimes in microcode, was necessary to get the high performance. However, when this could be done, the cost effectiveness of this type of hardware was impressive. Enrico Clementi of IBM-Kingston was able to perform large computations by running on multiple FPS 164 s controlled by an IBM mainframe. This success led Cornell University to create a high-performance parallel system by attaching two FPS 164 and five 264 processors to an IBM $3090-400$ computer.

Another successful wide instruction computer is the ST-100 array processor. With a 40 nanosecond clock cycle and four independent programmable processors, its peak performance is around 100 Mflops in single precision (32-bit) arithmetic. A separate processor is dedicated to each of the following functions: external data flow, internal data flow, arithmetic processing, and synchronization. Arithmetic processing is performed by 32-bit floating point arithmetic, pipelined functional units: two adders, two multipliers, and a 480 nanosecond divide/square root functional unit. Several memory references and logical operations and four arithmetic operations may be started in each machine cycle. Since it does not support 64-bit floating point arithmetic and at present has no compiler for a high level language, it cannot be considered a general puipuse scientiflc computer. Nevertheless it provides evidence that this type of architecture can be cost effective; with careful programming it has achieved a sustained performance two-thirds that of a Cray X-MP processor for important scientific computations like QCD.

The latest commercial wide instruction word machines carry that architectural trend much farther. The CHoPP by Sullivan Computer will have a 256-bit "superinstruction" that is equivalent to nine instructions on a conventional system. Four functional units can perform address computations while four other functional units can perform full instructions, including floating point arithmetic. The ninth unit is reserved for branching. The CHoPP will be 
available in configurations of four to sixteen CPUs with a large shared memory. Peak performance for the four CPU configuration is projected to be 270 Mflops; simulated performance for Livermore Loops $1-14$ is 81 Mflops.

The Multiflow computer uses an instruction word that can be as wide as 1024 bits. Each instruction can start as many as twenty-eight primitive operations on reduced instruction set processors. Orchestration of the actions of that many low-level processors requires a highly sophisticated compiler. When it is successful, parallelism can be exploited at a very fine level. Other than the sophistication of the compiler technology, the software environment is conventional. Compilers for Fortran and $C$ are provided. The UNIX operating system and TCP/IP networking protocols are used. Performance data are not yet generally available. At the time this paper was written, the first Multiflow computer was undergoing field tests. The smallest Multiflow system, the TRACE 7/200 which has a 256-bit instruction word, reached 6.0 Mflops on the full precision LINPACK benchmark. For a C program that does symbolic manipulations, but no floating-point arithmetic, it ran 16 times as fast as a VAX 11/780.

\section{Lattice Connected Machines}

SIMD machines consisting of large numbers of lattice-connected bit-serial processors constitute a high-performance advanced architecture that has been in use for some time yet can still be regarded as advanced. The Goodycar Massively Parallel Processor (MPP), the International Computers Limited (ICL) Distributed Array Processor (DAP), and the recently announced DAP-3 all fall in this category. They are similar to the Connection Machine but have a lower-dimensional connection network among processors.

The ICL DAP has been available since the late 1970's. Half a dozen machines have been installed. As was mentioned earlier, the DAP is an SIMD lockstep machine that operates on multiple data a bit at a time. Through programming, arithmetic can be carried out in variable precisions. The processing elements form a grid with nearest neighbor connections. 
Typical configurations have a 64 by 64 grid of processors, each with 2048 bytes of memory. An operation can be performed on each processing element at each clock cycle of 200 nanoseconds. Masking enables or inhibits execution of the (same) instruction for each processor. Fortran is available through a cross-compiler that runs on the host ICL 2900 . A 32 by 32 version of the DAP; designated the DAP-3, has been announced by Active Memory Technology Inc. of Atlanta, Georgia. Memory sizes range from $64 \mathrm{~K}$ to one megabit per processor. As of this writing, none has been installed.

Goodyear Aerospace Corporation manufactures the Massively Parallel Processor (MPP). The only MPP built to date was delivered to NASA's Goddard Space Flight Center in May 1983. Its 16,384 processing elements are bit-serial processors arranged in a 128 by 128 grid with nearest-neighbor connections. Until the Connection Machine CM-2 was announced, the MPP was the highest performing SIMD system available. For image processing tasks it has performed over one billion operations per second. In numerical computations like solving partial differential equations, hundreds of Mflops have been achieved. The MPP utilizes Parallel Pascal as its high-level language.

The British company Inmos produces the Transputer, a 7.5 MIPS chip with four communications channels built-in, which make it well suited as a building block for latticeconnected systems. A new version with 1 Mflop performance will be available in September. Both Inmos and another British company, Meiko, market Transputer-based systems. It is expected that the University of Edinburgh will soon get a system with 1024 nodes of the new version. That configuration would have over 1 Gflop peak performance and 2 Gbytes of memory.

\section{Other Architectures}

BBN Advanced Computers Inc. produces the Butterfly family of computers. These systems consist of microprocessors and memory units that are connected to each other via a 
specially-designed switch. Although all memory is local to the processor to which it is attached, each processor has access every other processor's memory through the switch. This general type of connection scheme is found in several research computers, such as the NYU Ultracomputer and IBM's RP3, but at present is unique to the Butterfly among commercial systems. Butterfly processor nodes consist of Motorola 68000 or 68020 microprocessors with one to four megabytes of memory. The largest configuration built to date has 256 processors. On that system typical numerical algorithms like matrix multiply have achieved speed-ups as high as 234 , for an efficiency of 0.91 . A future system known as the Monarch is under development as a DARPA-sponsored research project. This system could have as many as 8,192 processors. The first configuration is expected to have 1024 processors, each capable of one MIP and one Mflop. Fortran, C, and LISP are available.

There is at least one commercial computer that uses a dataflow architecture, the Loral Dataflo. Its maximum configuration is 256 nodes. At each node are two National Semiconductor NS32016 microprocessors, one for data management and one for program execution. The latter has an NS floating point co-processor. There is shared memory as well as memory local to each node. The system is programmed by supplying a data graph description plus a graph node program written in Fortran or C. The grain size for the system is said to be approximately the size of a procedure, say 60 to 100 lines of source code. Little is known aboul performance of this system; there may not be any customer sites yet. It is clear that the present generation of this system cannot have very high performance. The microprocessors used are quite slow; even the maximal 256-node configuration would have a peak speed of about 10 Mflops with $100 \%$ efficiency. It is included in this paper primarily because of its unusual architecture for a commercial computer.

The CYBERPLUS computer built by CDC is another unusual system. It is a multiple parallel processor system with a ring bus architecture. This computer features an 800 Megabits/second transfer rate with a read and a write possible between processors at this sustained rate. Two CYBERPLUS processor models are available: 16-bit integer and 64-bit 
floating point. The floating point processor has a peak performance of 65 Mflops in 64-bit mode. Each processor has a 20 nanosecond cycle time. There are fifteen independent functional units and it is possible to start several operations in each instruction. Thus it has a wide instruction word architecture as well. Up to 16 CYBERPLUS processors can be connected to a ring. Within one ring all processors operate autonomously and may execute each clock cycle. Processor Memory Interface allows direct reading and writing of the memory of any processor by another processor on the ring every machine cycle. Up to 16 rings can be connected to a Cyber 800 series host computer. There are three distinct memory systems of different sizes and speeds. The host CDC computer (using the NOS 2 operating system) loads code into the processors, transmits data from host to processors, and starts and stops each processor's task. Software includes a cross assembler and an ANSI 77 Fortran cross-compiler. Notwithstanding the richness of the CYBERPLUS architecture, it has not established itself as a powerful general-purpose numerical computer. The peak performance of a large CYBERPLUS configuration is very high: 64 CYBERPLUS systems linked together are claimed to perform 16 Gflops (probably 32 bits) on a signal processing application. However, the existing customer sites have not been able to reach a substantial percentage of that performance for scientific computations.

\section{Software Environment}

\section{Languages and Compilers}

There is a greater variety of languages and compiler technologies in the emerging supercomputers than in the traditional ones. Although most systems have (or plan to add) a Fortran compiler, examples of the diversity are the use of Occam in the FPS T-Series as the high-level language; special versions of LISP and C for the Connection Machine; and the existence of C and Pascal compilers in most systems.

The Fortran language for these systems is generally augmented by special syntax, system routine calls, or specially coded comments that provide additional information to the compiler. 
In large-grain machines, compilers seldom aid in the parallel programming task. An exception is the Alliant's Fortran compiler that does automatic parallelization as well as automatic vectorization. Its ability to detect automatically vector operations and parallelism and to generate efficient code is respectable.

In contrast, compilers for fine-grain computers like the Multiflow and the Connection Machine perform a great deal of the parallelization automatically and are crucial to the performance achieved on those systems.

\section{Operating System and Networking Trends}

Virtually all the new high-performance computers (or their hosts) have some version of the UNIX operating system. In many cases the advanced system itself has a minimal operating system, but requires a host that runs UNIX. There are some exceptions to this pattern. The Connection Machine uses a Symbolics LISP machine as a host, though even here support for a VAX/UNIX host will soon become available. The DAP-3 can be hosted by a VAX running VMS, as well as a VAX or Sun running UNIX. The FPS array processors can be hosted by an IBM/MVS front-end or a VAX/VMS front-end, as well as UNIX systems. The FPS TSeries is hosted by a micro-VAX with the VMS operating system. The ST-100 can be hosted by VAX/VMS, micro-VAX, Gould, and Perkln-Elmer systems. The NCÚBE system, since it requires no external host, runs most of the operating system on its Host Board with parts of the operating system running on the nodes. The NCUBE operating system, called AXIS, is similar to UNIX.

Since in most cases the advanced architecture machincs have a host that runs a variant of UNIX or VMS, TCP/IP and DECNET networking protocols are generally available, with the former being the most common. 


\section{Application Software}

There is limited availability of mathematical software, graphics libraries, and application software (such as engineering packages) for the advanced architecture computers. This is understandable since so few copies of any model have been installed for production use. For medium performance systems like Sequent, Encore, and Alliant, mathematical software libraries are under development. Sequent and Encore have begun to offer database packages. Engineering application packages are generally not available. This lack of application software is a significant obstacle to the use of the new systems in a supercomputer facility for a general user community. It is less important for basic research that requires supercomputing capability. For these uses, the programs are often written entirely by the research group responsible for the computation.

\section{Current Use of Advanced Architecture High-Performance Computers}

Computers with advanced architectures, as defined in this paper, are being used to perform large computations. A few advanced architecture systems have achieved performance on real user programs that is high enough to approach supercomputer levels but no highly parallel machine is in use as a supercomputer in the traditional sense. The Goodyear MPP is probably the closest to that status, but to achieve good performance on the MPP requires a substantial effort by the user and frequently the use of assembler language.

In most cases, the machines that are the topic of this paper do not exist in large enough configurations to be considered supercomputers. Furthermore, they were typically acquired for exploratory investigations on the use of parallel architectures. As a result, there are few systems that are used as workhorses for large-scale, production applications. On the other hand, the experience to date is encouraging for those who expect to obtain highly cost effective and high performance computing resources through adyanced architectures. 
It is interesting to note that the chief cause of the moderate performance of today's advanced architecture computers is the modest power of the processors used, rather than inability to use the systems with high efficiency. As was noted earlier, speed-ups of 234 have been measured on a 256-processor Butterfly. The SIMD fine-grain systems manage to achieve extremely high degrees of concurrency. Thus it appears possible that well-balanced, highly parallel architectures will indeed become supercomputers when they incorporate individual processors with higher performance than those currently used or, in the case of fine-grain SIMD machines, when hundreds of thousands or millions of processors are used.

The experience data presented here are necessarily sparse and vague; it is intended to give a feel for what has been achieved, rather than a systematic survey of all use of innovative systems. As was mentioned in the description of the NCUBE, William Martin at the University of Michigan has done Monte Carlo photon transport computations on a 64-node NCUBE at speeds that were a substantial fraction of a Cray X-MP-48. On a 16-node Intel iPSC-VX system, a system of 1000 dense linear equations was solved at a rate of 11 Mflops. On a seismic modeling application, performance of 227 Mflops (32 bits only) was achieved on a 32node system with vector processors, but microcoding was used. With the new version of the iPSC and a 64-node configuration of the iPSC-VX, speeds of over 100 Mflops should be possible. An Intel iPSC 32-node system (but without the vector boards) is being used to process seismic data at the Christian Michelsen Institute in Bergen, Norway. The DAP's main applications are in lattice gauge theory and molecular dynamics. It is particularly powerful on the Ising model because of its bit arithmetic. It is also used in many Monte Carlo calculations and in image processing where the major problem is in data movement rather than processing speed. For some specialized applications, the DAP will outperform a Cray-1.

In brief, as was mentioned at the beginning of this section, this author is not aware of any supercomputer facilities that utilize systems with the novel architectures described in this paper. This situation may change in the near future as systems mature further, techniques for their use become more widely known, and a few institutions act as pioneers. 
At least two institutions have plans to use highly parallel architecture computers for supercomputer-level work: Cornell and Caltech.

Cornell University has announced intentions of creating a massively parallel supercomputer facility. Indeed the IBM/FPS computer combination that is in place now is being used for large-scale scientific computing. Scientists from many disciplines have used the system to carry out major computations. The configuration consists of a conglomerate of commercial systems that provide a modest level of concurrent processing capability, rather than a massively parallel, unified architecture: the IBM 3090s provide four vector units and mainframes to control and host the various FPS array processors.

The California Institute of Technology has been using hypercubes for scientific computations since 1983. The Caltech-designed and built Cosmic Cube and Mark II systems have been used for $\mathrm{QCD}$ computations that have led to a number of physics publications, even though those systems are much slower than supercomputers: the 128-node Mark II runs at about 5 Mflops. This was possible because the machines could be dedicated to one computation for long periods. Scientists from other disciplines have also used the Caltech hypercubes for their work. In addition to the locally built systems, commercial hypercubes from Intel and NCUBE have been acquired and used by Caltech. In collaboration with the Jet Propulsion Laboratory (JPL) the Mark III hypercube has been built and a version with high-speed floating point, the Mark IIIfp, is under construction. When the new tloating point version is completed, the 128-node Mark IIIfp will have a peak speed of over one Gflop. The NCUBE system will be expanded to 512 nodes the summer of 1987 and possibly 1024 nodes later. These systems form the hardware basis for the recently formed Concurrent Supercomputer Initiative at Caltech (CSIC), a project whose goal is to create a supercomputer facility based on highly concurrent architectures. 


\section{Limits of Scalability}

How big a computer can be built and used in the next three to five years? This question is asked increasingly often as large-scale computing pervades more and more disciplines and practitioners of those disciplines. Scientists and engineers always seem to need several orders of magnitude more computing power than is available. How likely is it that in the next three to five years it will be possible to provide an increment in computing power of two or three orders of magnitude? Earlier in this article current supercomputers were characterized as having a peak speed of a few Gflops and an attainable speed of several hundred Mflops. Memory sizes range from hundreds of megabytes to a couple of gigabytes. An increase in speed of two to three orders of magnitude would result in systems with a peak speed of 1,000 Gflops and attainable speeds of 100 Gflops or more. If memory sizes kept pace, these systems would have directly addressable memories of tens to hundreds of gigabytes. Can such systems be built in the near future? What type of architecture might they have? If they incorporate massive parallelism, is there any hope that they can be programmed effectively, so that single large scientific computations could use them efficiently? These are difficult questions to answer conclusively, but some rough estimates can be made with a simplified analysis of the construction and usability issues. We will restrict the analysis that follows to traditional scientific computations that are dominated by floating-point operations.

A widely accepted estimate of further speed increases for a single sequential processor is only one order of magnitude more than the fastest currently available. That estimate is based on advances in device and chip technology. Given that some systems currently have cycle times of as little as four nanoseconds, cycle times of a few hundred picoseconds would be possible. Computers built with such technology are surely more than three years away, since devices of that speed are not yet available as commercial products. We assume that in the near future device speeds for the fastest chips available will increase at most by a factor of four, and therefore will not be the key to getting two to three orders of magnitude speed increase. 
We also assume that tying together 1,000 vector processors each with a peak speed of 1 Gflop is not a realistic way to create a system with a peak speed of 1,000 Gflops. Even if one used a distributed memory approach with a simple connection scheme such as ring topology, the cost would be prohibitive. Vector processors that run at $1 \mathrm{Gflop}$ are expensive; the communications channels to link them would also be expensive. If the cost per vector processor were reduced to $\$ 1$ million and communication channels were cheap, it is still unlikely that any institution would spend over $\$ 1,000$ million for the system. The physical size of such a configuration would require a large building as well. This naive analysis suggests that in the near term one would have to use large numbers of much smaller and cheaper processors to build a very high performance system. For example, the new XL-series floating-point and integer arithmetic chip set is just becoming available from Weitek. At present only 32-bit versions are in production; early next year the 64-bit versions will be available. These chips have a peak speed of over twenty Mflops. As was mentioned in the description of the Mark IIIfp hypercube with Weitek chips, speeds of 16 Mflops have been measured on hand-coded assembler code to multiply two $3 \times 3$ complex matrices. Five Mflops ought to be possible on a reasonably wide range of computations. Other vendors may have or are developing chips with similar or better performance. In keeping with the style of this article, the Weitek chip set is used as an example and proof of existence, not as an indicator of the most advanced commerrial product available.

How fast a system could one build today with such components? It would require 50,000 such units to reach a peak speed of 1,000 Gflops. Can systems that big be put together? There are indications that they can be, at least if a distributed memory architecture is used. The Connection Machine Model 2 has 65,536 bit-serial nodes and 2048 floating-point units (based on a different Weitek chip set). This system is not very big physically. Layouts for configurations with sixteen times as many processors have reportedly been worked out. Based on these considerations, it would appear possible to build a system with 32,768 floating-point units. If each has 20 Mflops peak performance, the system peak speed would be 
655 Gflops. Based on early experience with the XL-series Weitek chips, well over 100 Gflops would be attainable for real applications on such a system. Using 5 Mflops as an estimate of achievable performance, a $32 \mathrm{~K}$-processor system would produce over 150 Gflops for highly parallelizable computations. If the price and size of this system were directly proportional to existing models, it would be realistic to build it.

The analysis above was based on just one possible system. Other components and connection schemes should also reach the $100-1,000$ Gflop goals. Faster components are certain to be developed in the near future. In summary, it appears feasible to build systems with much higher performance.

Much larger memory sizes are also attainable, provided one can use relatively slow memory. In distributed memory architectures, memories need only be well matched in performance with the local processor (or processors, in the case of cluster architectures). Sharedmemory architectures can also effectively use memory systems with components that are slow relative to the aggregate processor speed, provided a suitable pipeline or memory-access network is used. In shared-memory systems, once more than 4 Gbytes are used it will be necessary to devote more bits to addresses than at present. This is a cost, not feasibility, issue.

Having dealt with the hardware issues, admittedly in a most superficial manner, would it be possible to program systems with that much parallelism? Will the efficiency achieved be acceptable or will a variant of Amdahl's Law doom us to waste most of the hardware resources?

There is little doubt that it is possible to use effectively systems with a few hundred 32 or 64-bit processors or thousands of bit-serial processors. For typical scientific and engineering computations it has already been demonstrated that efficiencies of $70 \%$ or more can be achieved on systems of that type. The necessary algorithm redesign and re-implementation can often be done with moderate effort. High-level languages with some system subroutine calls are generally adequate; hand=coded assembler oi miçrucode is not required. 
Does the situation change for systems with thousands of 32 or 64-bit processors or millions of bit-serial processors? The answer depends on the computation to be carried out. For many scientific applications it appears feasible to use effectively arbitrarily large numbers of large-grain processors, provided the computation to be carried out is big enough. We will sketch out below the analysis and supporting evidence.

A number of real applications have been discovered to be "embarassingly parallel," that is, for sufficiently large problems, the computations can easily be decomposed to run on arbitrarily many processors with essentially no serial bottleneck. For such applications, the key question is whether it will be possible for each processor to get at the data it needs without incurring heavy overheads. On shared-memory systems this translates into whether one can design (and build at a reasonable cost) a processor-memory interconnection scheme that enables processors to get data from memory at a fast enough rate and without interference among themselves.

Shared-memory systems with thousands of processors have not yet been built. Research and development of effective interconnection networks is proceeding at several institutions but their near-term targets for the maximum configuration are at most 4096 processors. Even if massively parallel shared-memory systems are built, it is not known at this time how effectively the connection schemes, once built, will avoid bottlenecks.

For distributed-memory architectures, systems have been designed and in some cases built with many processors, so we assume that scalability of the hardware is not a key issue. Therefore, let us focus on a processor's ability to get the data it needs quickly enough. The communications channels among processors are typically slower than direct memory access but simpler to characterize, especially if there is no overlap of processing and communications. If the data are in the same node, i.e., in the processor's local memory, there should be no problem, assuming that the speed of the processor and memory at each node are well matched. With large memories at each node, the probability increases that the data are already in the node where they will be used. But there will be times when communication among nodes is 
necessary, either to transfer data or to synchronize activities. How big is the communications overhead? We summarize here two analyses, one by E. Amdahl and one by G. Fox.

In a keynote address at the Supercomputing '87 Conference, May 1987, "Tempered Expectations in Massively Parallel Processing and Semiconductor Industry," E. Amdahl presented an analysis of the scalability of parallel systems. There was no paper published in the proceedings, so what follows is based on notes taken during his presentation. Amdahl used the hypercube architecture as the basis for his analysis because from a hardware standpoint it is the most promising for extending without limit the number of processors. Here is my summary of his analysis. Consider an $N$-node hypercube, $N=2^{n}$. Let $W=$ the total (sequential) workload, let $c$ be the communication and task switching load among processors, and let $g$ be the "globality" of the data. The quantity $g$ reflects how often it will be necessary for a node to get data from another node. The case $g=1$, corresponds to random distribution of data, whereas if $g<1$ the probability that data are "far away" from the node that needs them decreases exponentially as $g \rightarrow 0$. Assuming that the average number of hops per communication is

$$
E_{A V}=\frac{g}{1+g} \log _{2} N
$$

and that one message per node is sent, then the workload on $N$ nodes, including communications, is

$$
W_{N}=W+\frac{c g}{1+g} N \log _{2} N
$$

i.e., it is the original workload plus the communication overhead. It is assumed that communications is not overlapped with computation; this is the case in most of the current commercial distributed-memory systems. Let $S$ be the speed of each processor. Then the execution time for one processor is 


$$
T_{1}=W / S
$$

and for $\mathrm{N}$ processors it is

$$
T_{N}=\frac{W_{N}}{N S}
$$

Restricting this analysis to the effects of communication, that is, assuming that the workload $W$ is $100 \%$ parallelizable, then the relative performance (speed-up) on $N$ nodes compared to one node is

$$
R_{N}=\frac{T_{1}}{T_{N}}
$$

or, substituting expression (1) for $W_{N}$ and simplifying,

$$
R_{N}=\frac{N}{1+\frac{c g}{1+g} \frac{N}{W} \log _{2} N}
$$

and the efficiency is

$$
\epsilon=\frac{1}{1+\frac{c g}{1+g} \frac{N}{W} \log _{2} N}
$$

Amdahl used equation (2) to conclude that one can get arbitrarily high performance on hypercubes, provided the workload is large enough. (In his presentation, the number of processors was used as the variable to adjust to get higher speed-up. I prefer to use grain size and total workioad as the variables; the results are the same.) What is needed is that $\frac{N}{W}$ grow more rapidly than than $\frac{N}{\log _{2} N}$, say

$$
\frac{W}{N} \approx N^{\delta-1} \frac{c g}{1+g} \log _{2} N, \delta>0
$$

Since $\frac{W}{N}$ is the workload per node, this is a measure of the grain size that must be used to get 
speed-up. According to equation (4), no matter how large $N$ is, one can get increased performance by adding more nodes, provided the problem is big enough that the grain size is suitable, that enough computation is done on each grain, and the communications load does not increase too rapidly with $\mathbf{N}$.

Amdahl pointed out that although performance can be increased indefinitely, the efficiency may be low. For example, if $g=1, N=1024$, and the communications workload $c$ is $0.1 \%$ as big as the computational workload $W$, then $\epsilon \approx 0.16$.

Amdahl also said that for certain computations efficiency could be kept high even for very large numbers of processors. He did not present the supporting analysis, but the following straightforward manipulations provide a basis for that statement. Clearly one wants the efficiency to be be as close to 1 as possible, even for very large $\mathbf{N}$. That corresponds to

$$
\frac{c g}{1+g} \log _{2} N \frac{N}{W} \leq \delta
$$

for some suitably small $\delta, \delta>0$. This will be true if

$$
\frac{W}{N} \geq \delta^{-1}\left(\frac{c g}{1+g}\right) \log _{2} N
$$

just as with speed-up, if the computational workload per node $\frac{W}{N}$ is large enough for the configuration used and the communications workload has a certain behavior. We need to characterize the type of workload for which (5) holds true. The left hand side of (5) can be made arbitrarily large by choosing a large enough workload $W$ for a given value of $N$ (or for a fixed workload choosing a suitably small $N$ ). Examine the right hand side of (5). $\delta$ is a constant and $\frac{g}{1+g}$ is always less than 1. The dominant behavior is that of $c \log _{2} N$, essentially the communications workload per node. If $c$ depends on $N$ and $W$, and grows with the grain size $\frac{W}{N}$ sufficiently fast, then (5) will not hold and efficiency will be low. Otherwise, for sufficiently large grain size, efficiency will be high. 
Fox $[4,5]$ had previously developed an analysis similar to that presented by Amdahl. The same qualitative results are obtained and empirical data are presented that support the conclusions. In addition, Fox noted that these results have implications on the memory size of each node and the amount of computation done on the data relative to the communication time required to transfer the data to the node where it will be used. Since this work has been published, only the key results will be be mentioned. In Fox's formulation, the following (machine-dependent) parameters are introduced

$t_{\text {calc }}-$ the time required to perform a generic operation

$t_{\text {comm }}-$ the time required to communicate one word from one node to another

For a given computation, the total calculation and communication per node are denoted

$T_{\text {calc }}$ - the number of operations done in each node $\times t_{\text {calc }}$

$T_{\text {comm }}$ - the number of words transferred to/from each node $\times t_{\text {comm }}$

The fractional communication overhead is defined as

$$
f_{C} \equiv \frac{T_{\text {comm }}}{T_{\text {calc }}}
$$

and it is shown that the efficiency can be expressed as

$$
\epsilon=\frac{1}{1+f_{C}}
$$

which is equation (3) with

$$
f_{C}=\frac{i g}{1+g} \log _{2} N \frac{N}{W}
$$

This is not a superficial similarity, because $\frac{W}{N}$ is a measure of the calculation time per node, which is what $T_{c a l c}$ represents, and $\frac{c g}{1+g} \log _{2} N$ was derived as an estimate of the communication time per node, similar to $T_{\text {comm }}$. Therefore we can rewrite (7) as 


$$
f_{C}=\frac{\frac{c g}{1+g} \log _{2} N}{\frac{W}{N}}=\frac{T_{\text {comm }}}{T_{\text {calc }}}
$$

so it follows that the results of Fox's analysis apply to Amdahl's model.

The crucial observation is that the fractional communication time can often be written in the form

$$
f_{C} \approx k \frac{t_{\text {comm }}}{F(m) t_{\text {calc }}}
$$

where $k$ is a constant, $m$ is a measure of the grain size (e.g., the number of points in the subgrid acted on by each node). Note that the machine parameters only appear in the ratio $t_{\text {cale }} / t_{\text {comm }}$.

Are there algorithms for which $f_{C}$ is small? Fox $[6,7]$ analyzes a number of scientific problems and particular algorithms to compute them and derives expressions for how much computation and communication is done at each node for a given value of $m$. For many algorithms, the function $F(m)$ is shown to be iudependent of $N$, the number of nodes. This implies that high efficiency can be attained on arbitrarily large systems. Some representative results are: 
Table 1

\begin{tabular}{|l|l|}
\hline $\mathrm{F}(\mathrm{m})$ & Problem Class \\
\hline $\mathrm{m}$ & $\begin{array}{l}\text { 1D Grid point Problems } \\
\text { Long range Forces }\end{array}$ \\
$\sqrt{m}$ & $\begin{array}{l}\text { Full or Banded Matrix LU } \\
\text { decomposition/eigenvalue determination } \\
\text { 2D Statistical Physics } \\
\text { Sparse matrices from 2D } \\
\text { finite element/difference }\end{array}$ \\
$m^{1 / 3}$ & $\begin{array}{l}\text { As above for 3 dimensions } \\
\text { Fast Fourier Transform }\end{array}$ \\
$\log _{2} m$
\end{tabular}

Recall that to get high efficiency $f_{C}$ must be small; when equation (9) holds, this is equivalent to having a large $F(m)$. This is true of all the entries in Table 1 that have a large enough grain size.

These algorithms and others have been implemented on several different hypercubes by Fox and his group. The actual performance and efficiency observed agreed well with the analysis presented above for systems with up to 128 nodes, the largest available at the time the studies were done.

\section{Summary}

The current trends in hardware suggest that it is realistic to establish a goal of several hundred gigaflops performance on a single system within three to five years, particularly if distributed memory architectures with large numbers of processors are used. Shared-memory systems may also scale to such performance, but there is less experience with massively parallel shared-memory architectures. Analyses by Amdahl and Fox, supplemented by empirical evidence, indicate that there are many computations that will achieve high efficiency on distributed-memory systems with very large numbers of processors. 


\section{Prospects and Conclusions}

There are many commercial products in the advanced computer architecture area. Many of the vendors are aiming at the super-minicomputer and mini-supercomputer market rather than aiming at supercomputer performance levels. The field is currently dominated by small companies that often have only one product line. It appears that most of the major manufacturers do not have products in the high-performance area that are based on new architectures, whether they be multiprocessors, wide-word architecture, or lattice. The exceptions are Floating Point Systems with its T-Series and Control Data with the CYBERPLUS system. IBM has the RP3 project for a system that would range from medium to massive parallelism, but that is a research project, not a commercial product. We are not aware of similar projects within Digital Equipment Corporation, ETA, Cray Corporation, UNISYS, and Gould, to name a few of the major manufacturers. Perhaps this is due to the relatively small size of the supercomputer market. Given that there are already half a dozen companies competing for supercomputer customers with "traditional" machines, there may be a feeling that it would be too risky to introduce radically new supercomputers. A second possible factor for the reluctance to develop advanced architecture supercomputers is the immaturity of the field. It is difficult to determine in advance whether a new architecture has high performance for a wide class of applications and is cost effective. Advanced architectures are filled with potential bottlenecks that dramatically reduce the performance that can be achieved.

The specific paths to high performance in today's commercial advanced architecture computers vary widely. Some, like the CHoPP, feature a few sophisticated and complex processors; others, like the Intel iPSC and NCUBE, utilize many microprocessors with a general-purpose instruction set; a few use very large numbers of extremely simple bit-serial processors. In all cases, high performance is gained through replicating components or processors, rather than through the use of new hardware technology that yields higher speed components. This is true of memory, bus speeds, and basic clock cycle times. On the other hand, 
software technology is playing an important role. For several systems, compiler techniques are the key to the feasibility of achieving high performance.

We are beginning to see the expected very wide range of performance that is possible in many of the parallel architectures. The FPS T-Series is an extreme example of this, but many of the others (existing or future) such as the CHoPP, the RP3, the ULTRAMAX, the CEDAR, the NCUBE, and the Intel hypercubes have a wide range of performance. cases the smaller configurations of a particular design are systems of modest performance but the biggest configurations reach fairly high performance. This property of the parallel systems should mean that with parallel architectures it will often be possible to have small to medium-sized systems owned by an individual or small group of people that are exactly compatible with true supercomputer systems, with the only difference being the number of processors in the configuration.

It appears that these innovative high-performance systems that are the topic of this paper are maturing rapidly enough in terms of hardware and software that in a very few yearsperhaps only two or three-we will see supercomputer facilities that feature one or more of these systems. These will be prototypical supercomputer facilities. Application software packages for engineering tasks, for example, will not yet be available. Optimizing and parallelizing compilers, program development tools, and debugging aids will probably still be less refined than the corresponding software for today's supercomputcrs, but they should be robust enough for use in real applications. It is important to note that even now several systems with the innovative architectures provide mini-supercomputer performance at an attractive price and, when dedicated to a small group of users, can tackle "supercomputer-sized" applications. 


\section{References}

[1] "Gigaflop Speed Algorithm for the Direct Solution of Large Block-Tridiagonal Systems in 3-D Physics Applications", D. Anderson, A. Fry, R. Gruber, A. Roy, UCRL-96034, Lawrence Livermore National Laboratory, January, 1987.

[2] "Microcanonical and Hybrid Simulations of Lattice Quantum Chromodynamics with Dynamical Fermions", D. Sinclair, ANL-HEP-PR-86-121, Argonne National Laboratory, October, 1986.

[3] "Monte Carlo Photon Transport on Advanced Computers", W. Martin, seminar presented at Argonne National Laboratory, January 8, 1987.

[4] "Matrix Algorithms on a Hypercube I: Matrix Multiplication", G.C. Fox, A.J.G. Hey, and S.W. Otto, Parallel Computing 4, p.17, 1987.

[5] "Solving Problems on Concurrent Processors", Geoffrey C. Fox, Mark A. Johnson, Gregory A. Lyzenga, Steve W. Otto, John K. Salmon, David W. Walker, to be published by Prentice Hall

[6] "Decomposition of Scientific Problems for Concurrent Processors", G. Fox, unpublished paper CALT-68-986, February, 1983.

[7] "Algorithms for Concurrent Processors", G. Fox, S. Otto, Physics Today, May, 1984. 